\title{
ON THE ORIGIN OF SOLAR RADIO NOISE
}

\author{
D. H. MENZEL AND M. KROOK \\ Harvard College Observatory, Cambridge, Mass., U.S.A.
}

The radio-frequency emission observed in solar bursts cannot reasonably be interpreted as thermal radiation. Its origin is to be sought for rather in terms of co-operative behaviour of systems of charged particles. In any case, we cannot avoid having to examine the physical consequences that arise from such co-operative behaviour.

Violent disturbances, involving considerable amounts of kinetic energy, are frequently observed in the lower layers of the solar atmosphere. These disturbances, initially localized, tend to be propagated through the atmosphere. Determination of the mode of propagation will depend on a study made within the framework of the dynamical theory of ionized gases. In particular, we must treat the material and the radiation fields on an equal footing as coupled systems. In general, the disturbance is propagated through the agency of the electro-magnetic field as well as through material motions. To account for noise bursts, we must discover physical processes that efficiently convert the available mechanical energy, by way of the propagation process, into electro-magnetic energy capable of leaving the boundary of the atmosphere as a radio wave.

We must, therefore, expect to treat the fundamental problem as dependent upon initial and boundary values in the dynamical theory of ionized gases. In constructing models for bursts, we expect the following factors to play essential roles:

(I) The non-linearity of the fundamental equations.

(2) The gradients of density, temperature, etc. in the solar atmosphere.

(3) The possible presence of magnetic fields.

We are still far from having a solution to any definite problem with specified initial and boundary values. The non-linearity of the equations leads to considerable complication in the theory. For this reason progress has practically been confined to investigating a few simple problems with a linearized form of the equations. In general, however, a disturbance, even when it has small amplitude in regions of high density, may grow to 
large amplitude in regions of low density. We can then no longer describe the phenomena even in terms of cross-modulation between solutions of the linearized equations. The non-linearity of the equations then becomes a very important part of the physical picture, as for example in the generation of shock waves.

It is, however, worth while to explore the consequences of the linearized theory as completely as possible. In view of the great extent of the solar atmosphere, we have to consider oscillations over the whole range from high to low density, i.e. from conditions where collisions predominate to conditions where collisions are negligible. It is extremely difficult to treat this whole range in a unified way on the basis of the Boltzmann kinetic equations. However, a unified treatment, in terms of a special model introduced by Bhatnagar, Gross and Krook, satisfactorily represents the effects of molecular collisions in gases. The model leads to kinetic equations which possess the same physical features as the Boltzmann equation but are mathematically more tractable. For small-amplitude oscillations, the distribution functions can be determined explicitly over the whole density range; dispersion relations can then also be determined for the whole range. Gross and Krook have applied the model to trace the continuous transition from sound waves at high density to longitudinal plasma oscillations at low density.

In the presence of magnetic fields the situation is much more complex. The mode of propagation depends on the relations between a number of parameters, e.g. collision frequencies, plasma frequencies, gyro-frequencies, etc. In general, longitudinal oscillations and transverse oscillations are coupled. In the limit of high density, transverse plasma oscillations take on the character of magneto-hydrodynamic waves; as the density tends to zero the oscillations goover continuouslyinto electro-magnetic wavesinfree space.

A mechanical disturbance in the lower layers of the solar atmosphere tends to excite longitudinal modes of oscillation of the medium. In the presence of a magnetic field, these oscillations in turn excite transverse modes. As these transverse waves penetrate into regions of lower density, their amplitudes tend to increase. At the same time the waves take on more and more the character of electro-magnetic waves. Under suitable conditions an appreciable fraction of the original mechanical energy may, in this way, eventually escape from the sun in the form of radio-frequency emission.

This research was supported in part by the Air Force Cambridge Research Center, Geophysics Research Directorate, through Contract AF 19(604)-1 394 with Harvard University. 


\section{Discussion}

Minnaert: Is there any explanation why the third harmonic has not been detected?

Menzel: Theory has not been refined to that point.

Pawsey: It would seem likely that the polarized bursts need an explanation in which a magnetic field is essential but the non-polarized bursts might have an explanation without a field.

Menzel: Our view has been that a magnetic field is necessary for transverse waves. But random fields would tend to randomize the polarization. 